\title{
Evanescent Wave Cavity Ring-Down Spectroscopy (EW-CRDS) as a Probe of Macromolecule Adsorption Kinetics at Functionalized Interfaces
}

\author{
Michael A. O’Connell, ${ }^{\dagger}$ Anne de Cuendias, ${ }^{\dagger}$ Florence Gayet, ${ }^{\dagger}$ Ian M. Shirley, ${ }^{\ddagger}$ Stuart R. Mackenzie, ${ }^{\S}$ \\ David M. Haddleton, ${ }^{* \dagger}$ and Patrick R. Unwin* ${ }^{* \dagger}$ \\ ${ }^{\dagger}$ Department of Chemistry, University of Warwick, Coventry CV4 7AL, United Kingdom \\ ${ }^{\ddagger}$ Syngenta, Jealotts Hill Research Centre, Bracknell RG42 6EY, United Kingdom \\ ${ }^{\S}$ Department of Chemistry, Physical and Theoretical Chemistry Laboratory, University of Oxford, Oxford OX1 3QZ, United \\ Kingdom
}

\section{Supporting Information}

\begin{abstract}
Evanescent wave cavity ring-down spectroscopy (EW-CRDS) has been employed to study the interfacial adsorption kinetics of coumarin-tagged macromolecules onto a range of functionalized planar surfaces. Such studies are valuable in designing polymers for complex systems where the degree of interaction between the polymer and surface needs to be tailored. Three tagged synthetic polymers with different functionalities are examined: poly(acrylic acid) (PAA), poly(3sulfopropyl methacrylate, potassium salt) (PSPMA), and a
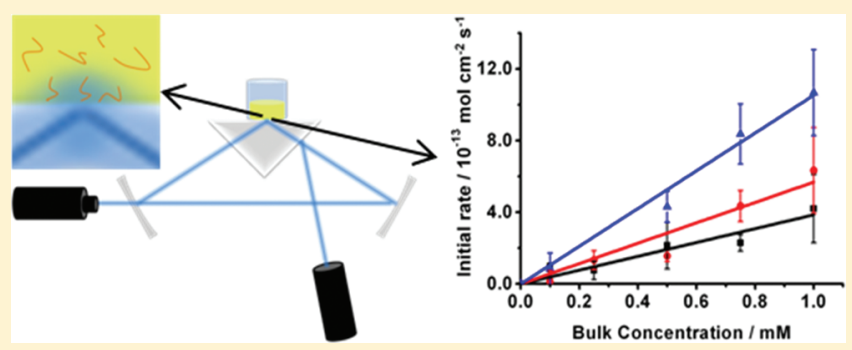
mannose-modified glycopolymer. Adsorption transients at the silica/water interface are found to be characteristic for each polymer, and kinetics are deduced from the initial rates. The chemistry of the adsorption interfaces has been varied by, first, manipulation of silica surface chemistry via the bulk $\mathrm{pH}$, followed by surfaces modified by poly(L-glutamic acid) (PGA) and cellulose, giving five chemically different surfaces. Complementary atomic force microscopy (AFM) imaging has been used for additional surface characterization of adsorbed layers and functionalized interfaces to allow adsorption rates to be interpreted more fully. Adsorption rates for PSPMA and the glycopolymer are seen to be highly surface sensitive, with significantly higher rates on cellulose-modified surfaces, whereas PAA shows a much smaller rate dependence on the nature of the adsorption surface.
\end{abstract}

\section{INTRODUCTION}

Understanding adsorption processes of both synthetic and biological macromolecules at interfaces is important for a great number of technologies and the development of new materials, spanning coatings, methods for colloid stabilization, reinforced plastics, and surfaces which are relevant to, or encourage, bioadhesion, among a wide range of possibilities. ${ }^{1-4}$ Fundamental studies of the adsorption of macromolecules, both in their free form and in micro- and nanostructures, are valuable for elucidating particular polymer properties that influence the mobility, adhesion, and ordering in polymer-adsorbed layers. ${ }^{5} \mathrm{~A}$ large variety of experimental techniques are currently used for such studies including the quartz crystal microbalance ${ }^{6-8}$ and a range of optical techniques such as ellipsometry, second harmonic generation, sum frequency generation, and Brewster angle reflectivity ${ }^{9-15}$ as well as theoretical studies, mainly using Monte Carlo and molecular dynamics approaches. ${ }^{16}$

Here, we demonstrate evanescent wave cavity ring-down spectroscopy (EW-CRDS) as a powerful approach to study the adsorption kinetics of polymers from bulk solution onto planar surfaces. First developed by Pipino et al., ${ }^{17}$ EW-CRDS is emerging as a key technique for the study of kinetic processes at solid/liquid interfaces. ${ }^{18,19}$ EW-CRDS combines the high sensitivity which arises from the multiple passes within a highfinesse optical cavity with the high spatial resolution resulting from evanescent fields. ${ }^{20}$ Evanescent waves are established whenever light undergoes total internal reflection (TIR) at the interface with an optically less dense medium and extend typically a few hundred nanometers beyond the interface. TIR usually occurs at the top surface of a fused silica prism with the evanescent wave formed penetrating a liquid-filled cell placed above this. A range of cavity geometries have been used to study various processes at the solid/liquid interface including interactions of dye molecules with silica ${ }^{21-23}$ and other organic molecules. $^{24,25}$ EW-CRDS and other related techniques ${ }^{26}$ are highly sensitive tools for studying changes at interfaces with sufficiently fast temporal resolution to study reasonably fast kinetics as exemplified by a recent review of applications. ${ }^{18}$

Although applications at liquid/air interfaces have been reported, ${ }^{18}$ most EW-CRDS studies to date have focused on

Received: February 10, 2012

Revised: April 9, 2012

Published: April 10, 2012 
silica surfaces; for example, Fan et al. used the adsorption of crystal violet cations to determine the relative populations of two different types of silanol groups at the silica surface. ${ }^{27}$ Initial EW-CRDS studies focused on the adsorption of small molecules on silica, with exemplar investigations of crystal violet, $^{23}$ Nile blue, ${ }^{22}$ methylene blue, ${ }^{28}\left[\mathrm{Ru}(\mathrm{bpy})_{3}\right]^{2+},{ }^{20}$ and rhodamine B. ${ }^{29}$ However, more recently the technique has been applied to more diverse problems, such as electrochemically induced desorption kinetics of silver nanoparticles, ${ }^{30}$ polyaniline nanoparticle formation and growth, ${ }^{31}$ and also bioanalytical applications. $^{32-36}$ The studies herein are the first on macromolecule adsorption and, furthermore, demonstrate further that the range of surfaces amenable to study can be diversified readily by chemical modification of the quartz prism.

The silica surface and silica/aqueous interface is much studied as it is common to many materials such as glasses, sands, and oxidized silicon, and as such has been wellcharacterized. Recent EW-CRDS studies ${ }^{21,23,27}$ have evaluated the complex surface chemistry of planar silica in detail using the crystal violet cation adsorption as a probe. As first reported by Ong et al., ${ }^{37}$ the silica surface exhibits two distinct types of silanol group: an isolated type with a $\mathrm{p} K_{\mathrm{a}}$ value of 4.9 and a surface population of ca. $19 \%$ and a vicinal type with a $\mathrm{p} K_{\mathrm{a}}$ value of 8.5 and surface population of ca. $81 \%$. The term "isolated" here refers to silanol groups unable to form hydrogen bonds with neighboring groups either directly or via bridging water molecules. The overall density of silanol groups on the silica surface is known to be $\sim 4.9 \times 10^{14} \mathrm{~cm}^{-2}$, giving $\sim 9.3 \times$ $10^{13} \mathrm{~cm}^{-2}$ isolated groups which can be further subdivided into type I and type II groups (based on degree of isolation) with respective densities of $\sim 1.05 \times 10^{13}$ and $\sim 8.2 \times 10^{13} \mathrm{~cm}^{-2} .38$ This knowledge of the surface chemistry and the ability to manipulate this by alteration of the solution $\mathrm{pH}$ makes silica an insightful surface to study the adsorption of complex macromolecules.

A further powerful feature of the EW-CRDS technique is the ease with which the silica surface can be modified with a variety of thin films and nanostructured materials such as polyelectrolyte films and bilayers. ${ }^{20,33,39,40}$ Indeed, the scope for functionalizing the prism surface is broad and encompasses any species that can be deposited onto silica (or subsequently onto layers previously adhered to the silica surface) provided it does not significantly affect the ring-down time. Herein we expand adsorption studies to chemical surfaces which are of broad interest and provide insightful comparisons with silica. One such surface chosen is cellulose, where depositions of regenerated thin films are well documented. ${ }^{41}$ As a polymer of $\beta$-glucose the surface chemistry is dominated by hydroxyl groups and provides an adhesion surface of great interest due to the presence in plant cell walls and the many industrial (cellulosic) derivatives, ${ }^{42}$ with an obvious area of interest being bioadhesion. An interesting counterpoint to this hydroxyl functionalization and silica surface chemistry is provided by an acidic functionality which can be formed trivially by the adsorption of high molecular weight polyelectrolytes, ${ }^{20}$ in this case in the form of poly(L-glutamic acid) (PGA).

The focus herein is the use of EW-CRDS to study the adsorption of three tagged polymers with different functional groups to a range of surfaces. We demonstrate EW-CRDS as an insightful and versatile tool and elucidate the adsorption kinetics of macromolecules as a function of bulk $\mathrm{pH}$ on silica and subsequently on modified surfaces. Characteristic adsorption transients are recorded for each polymer, and kinetic data (rate constants) are extracted for the initial part of the adsorption process.

Polymers exhibiting carboxylic acid, sulfonate, and sugar (mannose) functionalities were chosen for study in the form of poly(acrylic acid), poly(3-sulfopropyl methacrylate), and a mannose-modified glycopolymer, respectively (see Figure 1). A

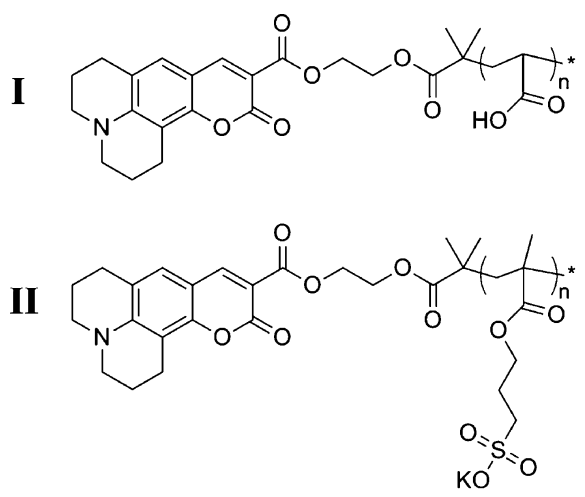

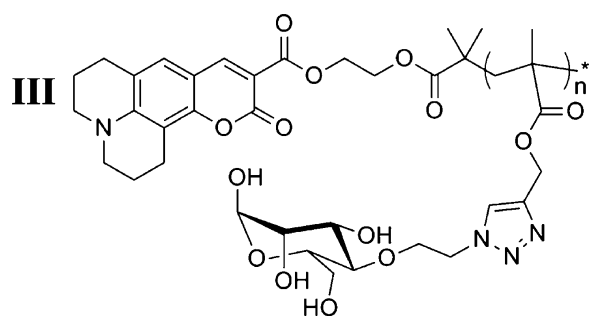

Figure 1. Structures of the coumarin-tagged synthetic polymers used in adsorption experiments.

single coumarin tag (incorporated as the initiator) served as an optical probe for each macromolecule, ensuring reasonable optical absorbance at the wavelength used $(405 \mathrm{~nm})$ in our EW-CRDS setup (see Figure S4, Supporting Information). The repeat units show respectively a single carboxylic acid side group, a sulfonate salt side group, and a mannose side group. In considering the adsorption of these molecules to surfaces, multiple binding routes are possible: the carboxylic acid chain gives a highly linear polyelectrolyte-type chain with minimal steric effects whereas the sulfonate group is larger and on a longer side chain. The glycopolymer displays the bulkiest modification with the multiple hydroxyl groups on the mannose ring facilitating multiple hydrogen-bonding opportunities. Both the sulfonate and glycopolymer will be little affected by bulk $\mathrm{pH}$, whereas the PAA will become increasingly protonated at low $\mathrm{pH}$. The studies herein allow an assessment of the impact on kinetics and extent of adsorption of the functionalities displayed by the macromolecules considered.

\section{EXPERIMENTAL SECTION}

Synthesis. Poly(acrylic acid) (PAA, I), poly(3-sulfopropyl methacrylate, potassium salt) (PSPMA, II), and mannose-modified glycopolymer (III) were synthesized as discussed briefly here and in detail in the Supporting Information: The coumarin initiator was synthesized via a two-step reaction by first producing an hydroxyethyl 2-bromoisobutyrate followed by DCC coupling with coumarin 343 . The coumarin tag was chosen as it absorbs reasonably at the $405 \mathrm{~nm}$ wavelength $\left(\lambda_{\text {abs }}\right.$ max at $443 \mathrm{~nm}$ in free form) of the diode laser used in our EW-CRDS setup. ${ }^{20,31,33,34}$ PAA (I) was synthesized via single electron transfer living radical polymerization (SET LRP) $)^{43-47}$ mediated with copper(0) and tris(2-(methylamino)ethyl)amine $\left(\mathrm{Me}_{6}\right.$-TREN) of tert-butyl acrylate $(t-\mathrm{BuA})$ with the coumarin 
functionalized initiator. The resulting $\mathrm{pt}$-BuA was deprotected by acidolysis with trifluoroacetic acid to give the desired PAA. PSPMA (II) was synthesized by the SET LRP of SPMA with the coumarin initiator mediated by $\mathrm{Me}_{6}$-TREN/copper(0). The mannose functionalized glycopolymer (III) was synthesized by the Huisgen cycloaddition of 2 -azidoethyl-O- $\alpha$-D-mannopyranoside ${ }^{48}$ with propargyl methacrylate, followed by copper(I)-mediated (ATRP) polymerization from the coumarin initiator. All reagents were obtained from SigmaAldrich unless otherwise specified. Full experimental conditions and schemes can be found in the Supporting Information. The structures of the resulting polymers are shown in Figure 1, and physical properties are summarized in Table 1. Molecular weights used for adsorption experiments were calculated by integration of the proton NMR peaks.

Table 1. Physical Characteristics of the Polymers Used in This Study

\begin{tabular}{lccc}
\multicolumn{1}{c}{ polymer } & $\begin{array}{c}M_{\mathrm{n}} / \mathrm{g} \mathrm{mol}^{-1} \\
(\text { from NMR) }\end{array}$ & $\begin{array}{c}\text { deg of } \\
\text { polym }\end{array}$ & $\begin{array}{c}\text { ext coeff at } 405 \mathrm{~nm} / \\
\mathrm{dm}^{3} \mathrm{~mol}^{-1} \mathrm{~cm}^{-1}\end{array}$ \\
I, PAA & 8100 & 105 & 4730 \\
II, PSPMA & 14000 & 54 & 539 \\
$\begin{array}{l}\text { III, mannose-modified } \\
\text { glycopolymer }\end{array}$ & 22200 & 58 & 10350 \\
\hline
\end{tabular}

Chemicals. Acetic acid, sodium acetate, sodium monophosphate, sodium diphosphate, sodium borate, and boric acid used for buffer preparation were bought from Sigma-Aldrich. Poly(L-lysine) (PLL, MW 30 000-70 000), poly(L-glutamic acid) (PGA, MW 50 000-100 000 ), and cellulose used for surface modification were also purchased from Sigma-Aldrich. All solvents used for cleaning of the prism surface were used at the highest purity available.

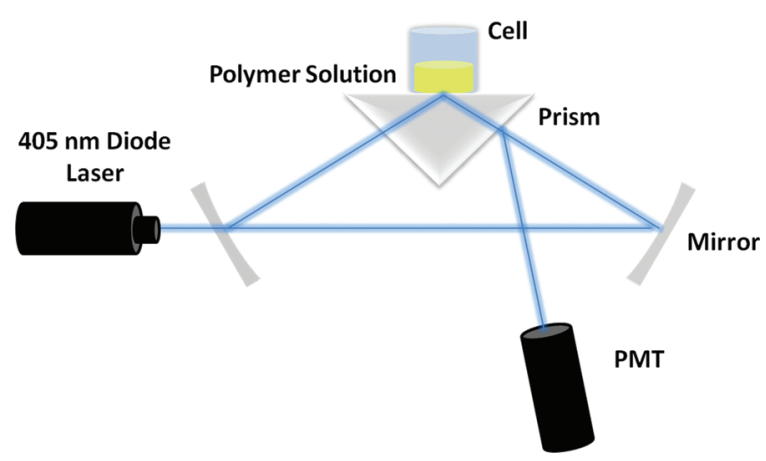

Figure 2. Schematic representation of the EW-CRDS setup used for adsorption experiments. PMT indicates photomultiplier tube.

EW-CRDS Apparatus and Adsorption Experiments. The EWCRD spectrometer (Figure 2) was operated in a ring-cavity geometry as described in detail elsewhere. ${ }^{30,49}$ The cavity comprised two highreflectance mirrors ( $R=99.997 \%$ at $405 \mathrm{~nm}$ (Los Gatos Research) and an intracavity right-angled fused silica prism (antireflective coated, $R<$ $0.5 \%, \lambda=350-532 \mathrm{~nm}$ for normal incidence (CVI Melles Griot)). Light pulses $(\sim 10 \mu \mathrm{s})$ were injected into the cavity using a TTL modulated diode laser $(405 \mathrm{~nm}, 50 \mathrm{~mW}$ maximum output, Power Technology Inc.). The light intensity circulating within the cavity was measured via the reflection from the incident prism surface using by a photomultiplier tube (Electron Tubes, 9781B). To make a ring-down measurement, the light source was rapidly extinguished $\left(t_{\text {fall }}<2 \mathrm{~ns}\right)$, and the PMT signal was recorded using a 12-bit $400 \mathrm{MS} / \mathrm{s}$ oscilloscope card (Gage CS12400). The ring-down time was calculated in real time using the fast Fourier transform method mo,49,50 $^{30}$ implemented within Labview.

A cell body was mounted on the hypotenuse of the prism and filled with polymer solution to create a solution/surface interface. The prism was wetted slightly prior to experiments to (a) allow optimization of the ring-down time and (b) ensure any adsorbed layers were fully hydrated. The characteristic ring-down time was measured as a function of time immediately after addition of the solution used. Data obtained (the characteristic ring-down time, $\tau$, as a function of time) were smoothed by plotting using 50-point adjacent averaging. The optical absorbance was calculated from the ring-down time using the equation $^{51}$

$$
A=\log _{10} e \frac{L}{c}\left[\frac{1}{\tau}-\frac{1}{\tau_{0}}\right]
$$

where $A$ is the optical interfacial absorbance, $L$ is the length of the cavity, $c$ is the speed of light, $\tau$ is the ring-down time, and $\tau_{0}$ is the initial ring-down time (typically for the situation where there was no adsorption at the solution/surface interface of question). The surface coverage due to adsorption was calculated by dividing the optical absorbance by the extinction coefficient (molecular absorption coefficient) derived from UV-vis spectroscopy (Lambda 25 UV-vis spectrometer, Perkin-Elmer Instruments; see Supporting Information Figure S4 for spectra and Table 1 for values). Note that the extinction coefficient varied widely between macromolecules with II considerably lower than I and III, with III retaining the strongest coumarin peak ( $\lambda_{\text {abs }} \max$ at $443 \mathrm{~nm}$ in its native form), i.e., the least affected by polymerization. Absorbance due to bulk solution appears as an instantaneous initial change in $\tau$ upon introduction of polymer solution into the cell and is easily subtracted.

Between each experiment the prism was wiped with methanol, treated with oxygen plasma for $3 \mathrm{~min}$ at $100 \mathrm{~W}$ (Emitech, K1050X), and wiped again with methanol. This resulted in the prism being returned to a clean state, as confirmed periodically by recording the background ring-down time, $\tau_{0}$, for a clean prism surface. Solutions were buffered at $\mathrm{pH} 4.8,7.0$, and 9.0 using solutions of acetic acid/ sodium acetate, sodium monophosphate/sodium diphosphate, or sodium borate/boric acid, respectively, all at $10 \mathrm{mM}$ concentration. All aqueous solutions were made using Milli- $\mathrm{Q}$ grade purified water (Millipore Corp.).

PGA Surface Functionalization. Poly(L-lysine) (PLL) solution $(1 \mathrm{mg} / \mathrm{mL})$ was drop-coated onto the top surface of the silica prism and left to adsorb for $20 \mathrm{~min}$. The surface was then rinsed with ultrapure water (Millipore) and dried under a stream of $\mathrm{N}_{2}$. Poly(Lglutamic acid) (PGA) solution $(1 \mathrm{mg} / \mathrm{mL})$ was then drop-coated onto the PLL-modified surface and left to adsorb for $20 \mathrm{~min}$, rinsed with ultra pure water, and dried under a stream of $\mathrm{N}_{2}$. A typical AFM image of a resulting surface is shown in Figure $3 \mathrm{~b}$ with the initial quartz surface (a) shown for comparison. This modification resulted in a small reduction in $\tau_{0}$ of typically $20 \mathrm{~ns}$ compared to the bare silica surface $(\sim 320 \mathrm{~ns})$.

Cellulose Surface Functionalization. Trimethylsilylcellulose (TMSC) was synthesized by the method described by Schaub et al. ${ }^{52}$ The silica prism surface was made hydrophobic by drop-coating with dichlorodimethylsilane and washing with copious amounts of propan-2-ol (IPA). TMSC was then dissolved in chloroform $(0.5 \mathrm{mg} /$ $\mathrm{mL}$ ), and the solution deposited dropwise onto the water surface of a Langmuir trough (NIMA Techology). The monolayer was compressed to typically $25 \mathrm{mN} \mathrm{cm}{ }^{-2}$, and layers transferred to the hydrophobic prism surface via the Langmuir-Schaefer technique. The TMSC multilayers were then converted back into cellulose by exposure to concentrated $\mathrm{HCl}$ fumes for $30 \mathrm{~s}$. The regeneration of cellulose was deemed to be complete following a change in the hydrophobicity of the surface, shown by the change in the static contact angle of water with the surface from $\sim 70-75^{\circ}$ for TMSC to $\sim 30-35^{\circ}$ after regeneration, which is in good keeping with literature values. ${ }^{53,54}$ Figure $3 \mathrm{c}$ shows the cellulose-modified surface comprising of five layers of cellulose which was found to give complete surface coverage while maintaining measurable ringdown times (i.e., $>50 \mathrm{~ns}$ ). This surface produced a larger reduction in $\tau_{0}$ of typically $\sim 100 \mathrm{~ns}$, leading to a small increase in noise levels on subsequent measurements. Nevertheless, as we show herein, convincing adsorption measurements could subsequently still be made readily. 


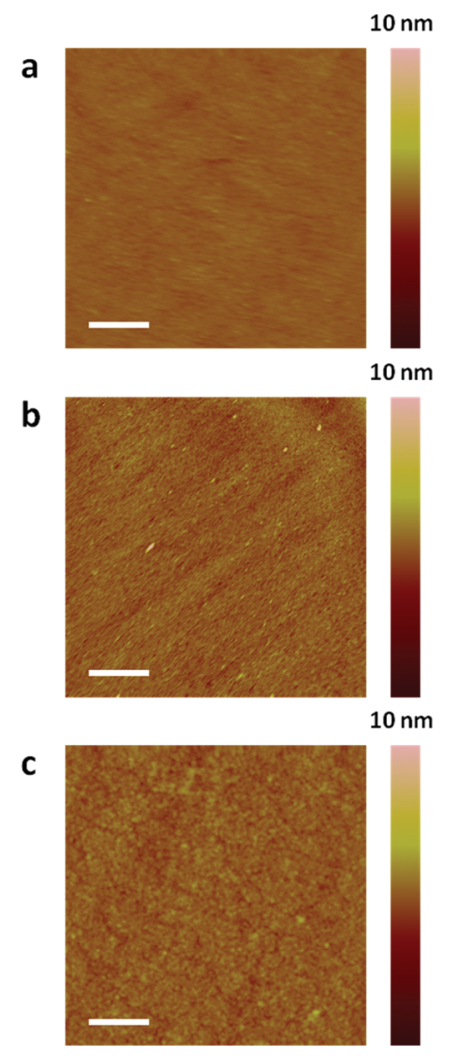

Figure 3. TM-AFM images showing (a) the bare silica surface, (b) PLL/PGA bilayer, and (c) regenerated cellulose thin film deposited onto silica surfaces. Scale bars denote $1 \mu \mathrm{m}$ for (a) and (b) and 200 $\mathrm{nm}$ for $(\mathrm{c})$.

Atomic Force Microscopy. AFM imaging was undertaken using a Bruker Multimode V AFM equipped with Nanoscope V controller operated in tapping mode (TM-AFM) using standard silicon tapping mode tips (RFESP or RTESP type, Bruker Probes). Polymer solutions and surface modifications (PLL/PGA and cellulose) were deposited onto highly polished polycrystalline quartz substrates (Hoffman Materials) which had been treated with oxygen plasma and wiped with methanol using identical conditions as for the silica prism (vide supra).

\section{RESULTS AND DISCUSSION}

Polymer Adsorption Experiments on Silica. EW-CRDS was first considered on the silica substrate, and adsorption was monitored over a time period of $1 \mathrm{~h}$. The adsorption vs time behavior of each polymer on silica was found to be characteristic. The EW-CRDS transient in Figure $4 \mathrm{a}(\mathrm{i})$ shows a relatively rapid initial rate of adsorption of $1 \mathrm{mM} \mathrm{I}$ at $\mathrm{pH} 7.0$ onto silica, which slows with time to give a limiting surface concentration (after $\sim 2500 \mathrm{~s}$ ) of $\sim 5.0 \times 10^{-10} \mathrm{~mol} \mathrm{~cm}^{-2}$. Note that this surface concentration, and all subsequent macromolecule surface concentrations, are defined in terms of tag concentration, i.e., that of an individual macromolecule, which contains a single tag with molecular weight based on $M_{\mathrm{n}}$ (from NMR). The shape of this transient appears to reflect simple adsorption behavior with vacant surface binding sites being occupied until a limiting value is reached. It is interesting to note that the limiting coverage in this case is similar to the surface density of silanol groups (vide supra). The relatively high number density of molecules at equilibrium could indicate close packing of macromolecules; this would be facilitated by the low steric demands of the PAA chain and the acidic groups forming a vertical polyelectrolyte brush, which is reasonable considering PAA is a weak polyelectrolyte $\left(\mathrm{p} K_{\mathrm{a}} \sim 4.5\right)$ which is essentially totally deprotonated at $\mathrm{pH} 7.0$ and the molecule studied has a fairly low $M_{\mathrm{n}}$ (of $8100 \mathrm{~g} \mathrm{~mol}^{-1}$ ). The corresponding TM-AFM image of the adsorbed layer deposited under identical conditions (Figure 4a(ii)) shows an apparently smooth layer with a low coverage of additional small particles on the surface. The particles visible are likely due to a small degree of aggregation of molecules which may partially explain the high (calculated) surface concentration of molecules. These observations (and those for subsequent macromolecules and surfaces) are discussed further in the kinetics section.

In stark contrast, polymer II again at $\mathrm{pH} 7.0$ shows approximately linear adsorption over the time period studied (Figure $4 \mathrm{~b}(\mathrm{i})$ ), meaning the observed initial rate of adsorption on silica is maintained at a constant value over a much longer time scale. Coincidentally, the surface coverage at $1 \mathrm{~h}(\sim 5.5 \times$ $10^{-10} \mathrm{~mol} \mathrm{~cm}^{-2}$ ) is of a similar order to that of $\mathrm{I}$, but there is no indication of an equilibrium being established. As there is no suggestion of preferential binding to particular sites on this surface, it can be assumed that the polymer has no preference for the silica surface and that multilayer adsorption occurs. AFM analysis of the adsorbed layer (Figure $4 \mathrm{~b}(\mathrm{ii})$ ) supports this, showing a continuous, smooth film with no indication of particles or aggregates on the surface.

Investigations of III were limited mainly by its lower (molar) solubility (due to its higher molecular mass). As such, concentrations an order of magnitude lower compared to I and II were used, i.e., $0.01-0.1 \mathrm{mM}$ compared to $0.1-1 \mathrm{mM}$. The adsorption transient in Figure $4 \mathrm{c}(\mathrm{i})$ shows an initially fast, but rapidly slowing, adsorption rate where equilibrium surface coverage is not reached after $1 \mathrm{~h}$. At all concentrations studied, III had not reached equilibrium surface coverage within this time scale, including for the same concentration as I $(0.1 \mathrm{mM})$ which would indicate the binding mechanism is either different, or on a much longer characteristic time scale, to that of $\mathbf{I}$. It is also clear from AFM analysis of the adsorbed layer (Figure $4 c(i i))$ that a large number of particles are present on the surface in contrast to I and II.

Adsorption Transients on PGA and Cellulose. Turning to cellulose and PGA, again at $\mathrm{pH} 7.0$, I shows similar adsorption vs time behavior (Figure 5a) to silica for both additional surfaces, except that on cellulose an apparent surface equilibrium has not been reached after the $1 \mathrm{~h}$ time scale. The equilibrium surface coverage for $1 \mathrm{mM}$ bulk concentration (I) at $\mathrm{pH} 7.0$ for both PGA and silica is similar at around $5.0 \times$ $10^{-10} \mathrm{~mol} \mathrm{~cm}^{-2}$ whereas surface coverage for cellulose is a little higher $\left(\sim 7.0 \times 10^{-10} \mathrm{~mol} \mathrm{~cm}^{-2}\right)$ and rising. Evidently the higher number of surface binding sites on cellulose compared to silica is a major factor (given the similar extent of adsorption).

Again in stark contrast, it is clear from Figure $5 b$ that the adsorption behavior of II is highly dependent on the surface, with a significant difference between PGA and cellulose, and the comparison on silica (Figure $4 \mathrm{~b}$ ). Adsorption on PGA shows a linear surface coverage vs time plot, as for the silica surface, but with a lower rate $\left(\sim 2.0 \times 10^{-10} \mathrm{~mol} \mathrm{~cm}^{-2}\right.$ after 1 h), whereas the cellulose surface results in an initial fast adsorption step followed by linear adsorption vs time behavior but also much more rapid adsorption $\left(\sim 1.3 \times 10^{-9} \mathrm{~mol} \mathrm{~cm}^{-2}\right.$ after $1 \mathrm{~h}$ ).

The adsorption vs time behavior for III on each surface followed the trend of rapid initial, but slowing, adsorption. 

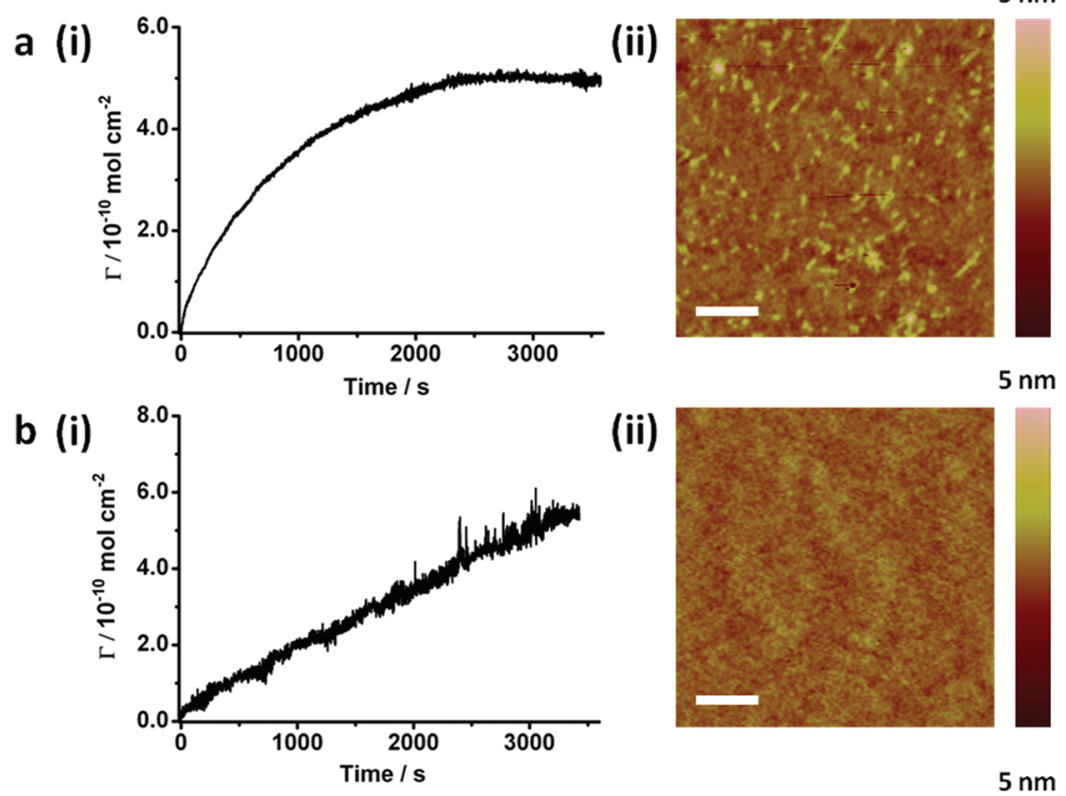

(ii)
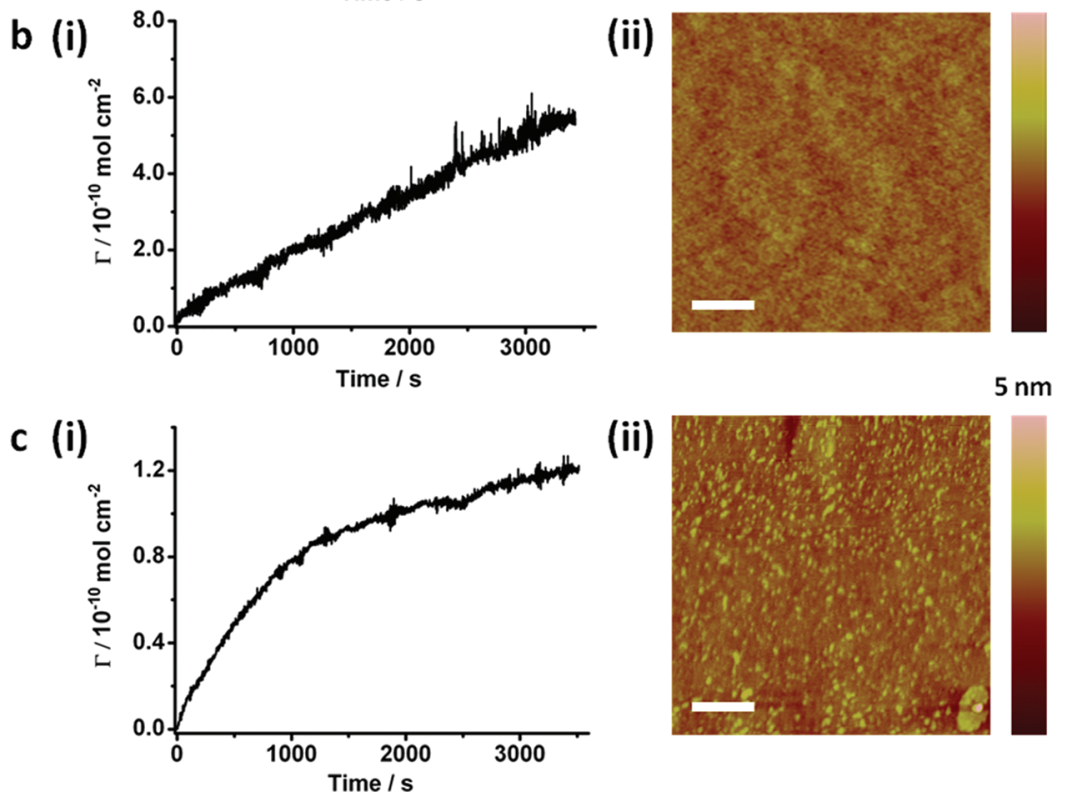

(ii)

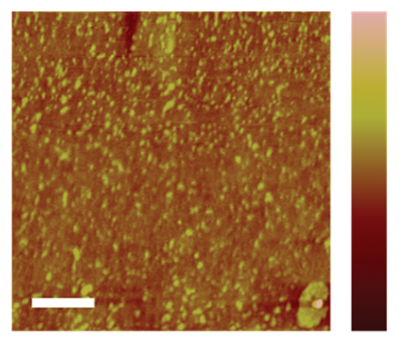

Figure 4. Typical EW-CRDS surface concentration, $\Gamma$, vs time plots at $\mathrm{pH} 7.0$ with TM-AFM characterization of the resulting surface: (a) (i) I at 1 $\mathrm{mM}$ concentration, (b) (i) II at $1 \mathrm{mM}$ concentration, and (c) (i) III at $0.1 \mathrm{mM}$ concentration. (a) (ii), (b) (ii), and (c) (ii) show the structure of the adsorbed layer. Scale bars denote $200 \mathrm{~nm}$.

However, it is clear that PGA significantly inhibited surface adsorption (by an order of magnitude), when surface concentrations after $1 \mathrm{~h}$ are considered, compared to silica and cellulose $\left(\sim 1.5 \times 10^{-11} \mathrm{~mol} \mathrm{~cm}{ }^{-2}\right.$ for PGA compared to $\sim 1.2 \times 10^{-10} \mathrm{~mol} \mathrm{~cm}^{-2}$ for silica and $\sim 1.3 \times 10^{-10} \mathrm{~mol} \mathrm{~cm}^{-2}$ for cellulose, respectively).

Reversibility. Desorption was also investigated with simple experiments to determine if the adsorption of each polymer to each surface was reversible. Adsorption was deemed to be reversible if, after removal of the polymer solution and introduction of buffer, the ring-down time returned (close) to its original value. The change was generally seen to be instantaneous due to the mechanical washing effect, and therefore no attempt was made to measure desorption rates. For consistency, the buffer was changed three times, and a return to within $10 \%$ of the original $\tau$ is deemed (reasonably) to be reversible. Irreversible adsorption was characterized by no significant change in $\tau$ on washing. Observations, which were found to be consistent across $\mathrm{pH}$ and concentration ranges, are summarized in Table 2.

Significantly, I was found to bind reversibly to all surfaces and III irreversibly to all surfaces, but II varied with surface; binding was reversible on silica and PGA, but irreversible on cellulose to which it adsorbed both more rapidly and in a nonlinear fashion (vide supra). The reversible binding of $\mathbf{I}$ provides further evidence that a surface equilibrium is reached, whereas for III this is clearly not the case despite a (superficially) similar adsorption vs time shape. Evidently, the sugar functionality promotes stronger surface binding. II provides an intermediate case; it is only irreversible on the hydroxyl-rich sugar polymer cellulose. However, the similarly negatively charged PAA (I) is reversible on this surface, with the ester linkage on the sulfonate side chain on II evidently providing sufficient $\mathrm{H}$-bonding with cellulose to cause irreversible adsorption.

Evaluation of Adsorption Kinetics. Data such as these in Figures 4 and 5 allow the initial adsorption rate $k_{\mathrm{i}}$, i.e., for $t<$ $100 \mathrm{~s}$, to be deduced. Focusing on this time scale is interesting because it reveals the adsorption kinetics on a fresh surface. Given the concentration used and time scale for adsorption, it is reasonable to assume the process is surface controlled; i.e., there are no diffusional effects. For first-order interfacial kinetics the following rate law thus holds:

$$
\left[\frac{\mathrm{d} \Gamma}{\mathrm{d} t}\right]_{t=0}=k_{\mathrm{i}} \mathrm{c}
$$

where $k_{\mathrm{i}}$ is an initial rate constant with units of $\mathrm{cm} \mathrm{s}^{-1}$. The initial part of each adsorption vs time transient at a series of different concentrations is thus fitted linearly to extract the initial adsorption rate, which is then plotted against bulk concentration to elucidate $k_{\mathrm{i}}$.

Effect of Bulk pH on Adsorption Kinetics to Silica. Rate constants were determined from plots of initial adsorption rate 

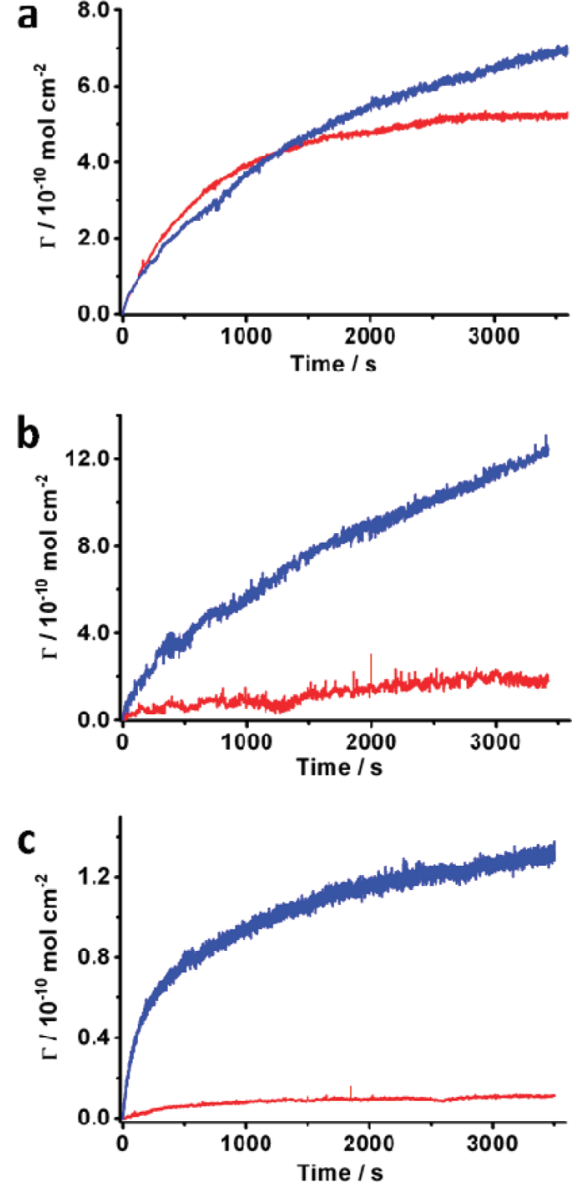

Figure 5. Typical EW-CRDS surface concentration, $\Gamma$, vs time plots at $\mathrm{pH} 7.0$ for PGA and cellulose for (a) I at $1 \mathrm{mM}$ concentration, (b) II at $1 \mathrm{mM}$ concentration, and (c) III at $0.1 \mathrm{mM}$ concentration. The transient obtained for PGA is shown in red and cellulose in blue.

Table 2. Observations of Reversibility Based on Washing of Prism Surface Postexperiment

\begin{tabular}{clll} 
polymer & \multicolumn{1}{c}{ silica } & \multicolumn{1}{c}{ PGA } & \multicolumn{1}{c}{ cellulose } \\
I & reversible & reversible & reversible \\
II & reversible & reversible & nonreversible \\
III & nonreversible & nonreversible & nonreversible \\
\hline
\end{tabular}

vs bulk concentration (Figure 6). $\mathrm{pH}$ values of 4.8, 7.0, and 9.0 were chosen to manipulate surface protonation levels (silanol groups with $\mathrm{p} K_{\mathrm{a}}$ values of 4.9 and 8.5). ${ }^{36}$ The linear fits of these plots indicate the assumption of first-order kinetics is reasonable. Rates for all kinetic experiments are summarized in Table 3 and plotted in Figure 7. The initial rate constants for I (Figure 6a) show a systematic increase in binding rates in the order $\mathrm{pH} 4.8<7.0<9.0$. The higher $\mathrm{pH}$ value promotes a faster adsorption rate especially when the silica surface is completely deprotonated ( $\mathrm{pH}$ 9.0). Naively, this might have been expected to lead to electrostatic repulsion between the surface and polymer molecules, suggesting that the intermolecular forces dominate the adsorption process on silica, with electrostatic repulsion between polymer chains driving polyelectrolyte brush formation, which eventually reaches equilibrium surface coverage at much longer time scales. Conversely, at $\mathrm{pH} 4.8 \mathrm{I}$ (as a weak acid of $\mathrm{p} K_{\mathrm{a}} \sim 4.5$ ) will become partially protonated, thus reducing electrostatic a

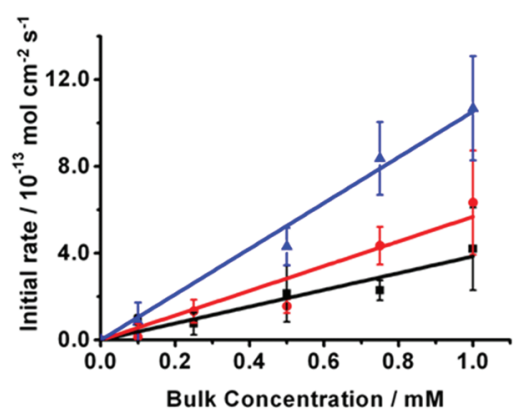

b

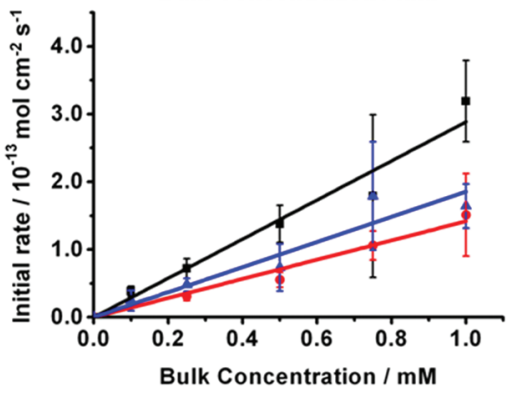

C

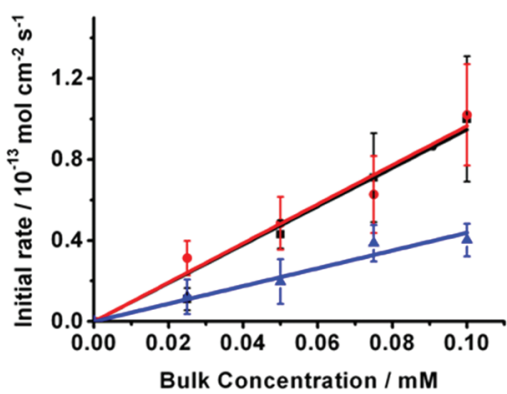

Figure 6. Kinetic plots of initial rate vs bulk concentration for (a) I, (b) II, and (c) III. The initial rate constant, $k_{\mathrm{i}}$, is equal to the gradient of the linear fit. $\mathrm{pH} 4.8$ is denoted in black, $\mathrm{pH} 7.0$ in red, and $\mathrm{pH} 9.0$ in blue.

repulsion (by lowering the charge density) between molecules and hindering (slightly) brush formation.

The initial adsorption kinetics for II (Figure 6b) show adsorption rates in the order $\mathrm{pH} 4.8>9.0>7.0$. These rates are all considerably lower than for I under the same conditions, highlighting that this polymer adsorbs more slowly at the silica surface at short time scales, as was evident when studying adsorption vs time behavior over a longer time period (vide supra). The slightly higher binding at $\mathrm{pH} 4.8$ is perhaps driven by less negative charge on the silica surface reducing electrostatic repulsion between the silica and sulfonate salt.

Initial adsorption kinetics for III (Figure 6c) were very similar for $\mathrm{pH} 4.8$ and 7.0 but around half that at $\mathrm{pH}$ 9.0. The mannose modifier on this polymer provides multiple hydrogenbonding opportunities and, coupled with the fact it is at the end of a comparatively long, bulky side chain, provides a likely binding route. This correlates with the decreased binding rate at $\mathrm{pH} 9.0$ as the surface exhibits the highest degree of deprotonation and therefore the fewest hydrogen-bonding sites.

Adsorption Kinetics on Modified Surfaces at pH 7.0. Figure 8 shows kinetic plots used to calculate $k_{\mathrm{i}}$ for PGA and cellulose surfaces in the same manner as in Figure 6 . The values are again summarized in Table 3 and Figure 7. The initial kinetics for I (Figure 8a) at $\mathrm{pH} 7.0$ show a marginally faster rate for cellulose compared to PGA. The effect of surface chemical functionalization on the adsorption rate of $\mathbf{I}$ is clearly minor, 
Table 3. Summary of Initial Rate Constants, $k_{\mathrm{i}}\left(\mathrm{cm} \mathrm{s}^{-1}\right)$, for All of the Surface Chemistries Studied

\begin{tabular}{|c|c|c|c|}
\hline & PAA (I) & PSPMA (II) & mannose-modified glycopolymer (III) \\
\hline silica (pH 4.8) & $(3.9 \pm 0.7) \times 10^{-7}$ & $(2.9 \pm 0.4) \times 10^{-7}$ & $(9.5 \pm 1.6) \times 10^{-7}$ \\
\hline silica (pH 7.0) & $(5.7 \pm 1.4) \times 10^{-7}$ & $(1.4 \pm 0.3) \times 10^{-7}$ & $(9.7 \pm 1.7) \times 10^{-7}$ \\
\hline silica (pH 9.0) & $(1.1 \pm 0.2) \times 10^{-6}$ & $(1.9 \pm 0.5) \times 10^{-7}$ & $(4.3 \pm 0.7) \times 10^{-7}$ \\
\hline cellulose ( $\mathrm{pH} 7.0)$ & $(7.8 \pm 0.7) \times 10^{-7}$ & $(2.8 \pm 0.6) \times 10^{-6}$ & $(3.4 \pm 0.4) \times 10^{-6}$ \\
\hline PGA (pH 7.0) & $(6.6 \pm 1.4) \times 10^{-7}$ & $(7.3 \pm 2.8) \times 10^{-8}$ & $(2.2 \pm 0.8) \times 10^{-7}$ \\
\hline
\end{tabular}

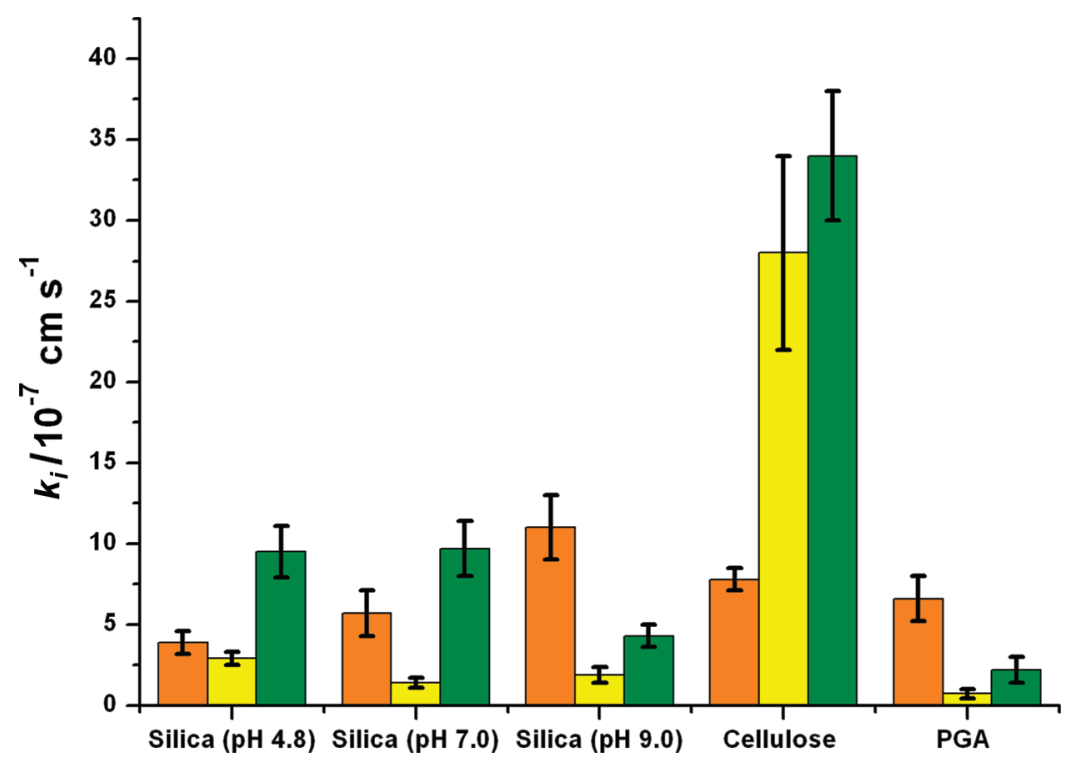

Figure 7. Summary of initial rate constants, $k_{\mathrm{i}}$, for all of the surface chemistries studied. I is shown in orange, II in yellow, and III in green.

which is consistent with the effect of manipulating bulk $\mathrm{pH}$ on silica (vide supra). The surface chemistries studied in this work clearly have little effect on the adsorption of I (PAA). Both the initial rates of adsorption and longer term studies give reasonably similar results across all surfaces and the conclusions made from the effect of manipulating silica surface chemistry hold for these further surfaces.

Figure $8 \mathrm{~b}$ shows the effect of surface functionalization on the adsorption rate of II. Here, the effect of surface chemistry on the initial rate is dramatic. The initial rate constant for adsorption to cellulose at $\mathrm{pH} 7.0$ shows a large increase compared to the silica reference (vide supra). Conversely, the PGA surface displays a significantly lower adsorption rate. These initial binding rates vary by a factor of 40 , which is in stark contrast to I. The lower rate on PGA can be explained by electrostatic repulsion between the negatively charged surface and sulfonate group on the polymer with virtually no binding sites available, whereas the hydrogen bonding between the polymer and cellulose massively increases this rate. In comparison to these the manipulation of silica surface chemistry has relatively little effect, though the most protonated surface (at $\mathrm{pH} 4.8$ ) does give the fastest rate of adsorption.

The initial adsorption rates of III (Figure 8c) show a similar, though less drastic, trend as II, with a clear increase in adsorption rate to cellulose and a decrease to PGA. The increase in adsorption rate to cellulose can again be reasonably considered to be due to increased hydrogen bonding, especially due to the multiple hydroxyl groups on the mannose pendant group. The deprotonated PGA surface will prevent this hydrogen bond formation and thus decrease surface adsorption, which is consistent with the data in Figure $6 \mathrm{c}$ where the lowest adsorption rate is for $\mathrm{pH} 9.0$.

\section{CONCLUSIONS}

EW-CRDS has been demonstrated to be a useful tool for probing the adsorption behavior and kinetics of macromolecules to planar surfaces. The silica surface inherent to this technique has been used as a model substrate and the surface chemistry manipulated by alteration of $\mathrm{pH}$, followed by chemical modification with polyelectrolyte and cellulose layers. Initial adsorption kinetics have been extracted, providing valuable information on binding rates.

For long time-scale experiments $(1 \mathrm{~h})$ adsorption vs time behavior on silica was found to be characteristic for each polymer. Initial adsorption kinetics were elucidated for each polymer as a function of $\mathrm{pH}$ on silica and extended to cellulose and PGA functionalized surfaces. For two of the polymers studied, PSPMA and mannose-modified glycopolymer, the adsorption kinetics were seen to be highly surface dependent with highest rates on cellulose-modified surfaces. PAA shows a much smaller rate dependence on the nature of the adsorption surface.

The studies herein highlight that the use of EW-CRDS for adsorption measurements at the solid/liquid interface holds much promise due to its relative simplicity and versatility. The ease of modification of the silica surface provides for many opportunities across a vast range of thin films and functionalities. Aside from simply studying adsorption/ desorption kinetics, the possibility to study further aspects of polymer adsorption such as exchange in polymer layers and effects under flow are also envisaged. 


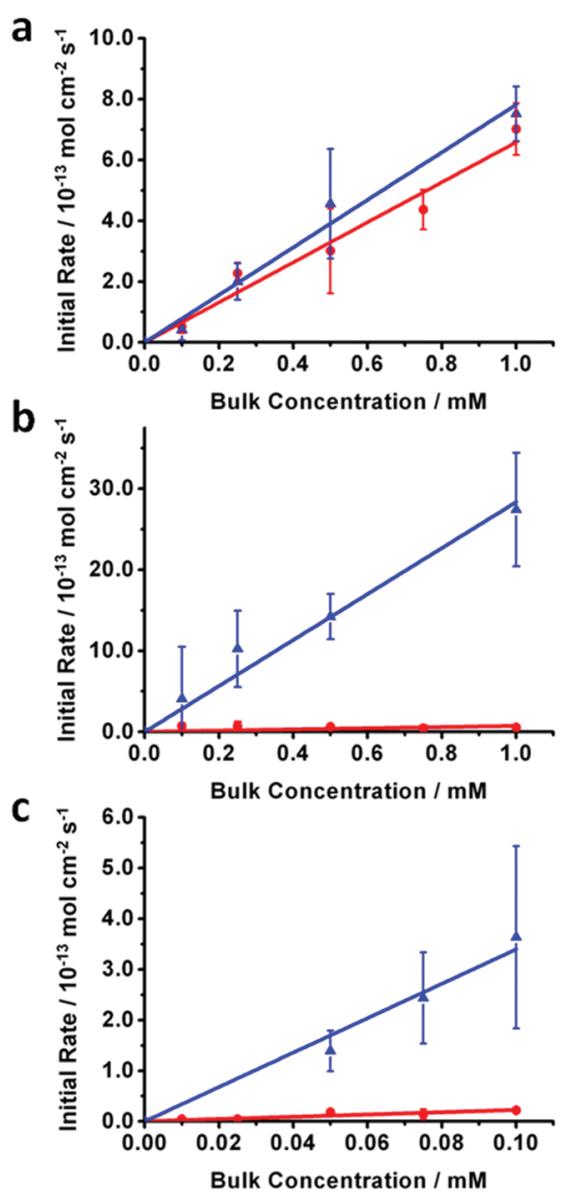

Figure 8. Kinetic plots of initial rate vs bulk concentration for (a) I, (b) II, and (c) III. The initial rate constant, $k_{\mathrm{i}}$, is equal to the gradient of the linear fit. PGA is denoted in red and cellulose in blue.

\section{ASSOCIATED CONTENT}

\section{S Supporting Information}

Full synthetic details, schemes, and analysis. This material is available free of charge via the Internet at http://pubs.acs.org.

\section{AUTHOR INFORMATION}

\section{Corresponding Author}

*E-mail: P.R.Unwin@warwick.ac.uk (P.R.U.); D.M. Haddleton@warwick.ac.uk (D.M.H.).

\section{Notes}

The authors declare no competing financial interest.

\section{ACKNOWLEDGMENTS}

This work was funded by the Engineering and Physical Sciences Research Council (EPSRC Grant No. EP/C00907X) and Syngenta (EPSRC CTA Studentship). The Multimode V AFM used in this research was obtained through Birmingham Science City: Innovative Uses for Advanced Materials in the Modern World (West Midlands Centre for Advanced Materials Project 2), with support from Advantage West Midlands (AWM) and part funded by the European Regional Development Fund (ERDF). The authors thank Andrew Edwards for help in synthesizing TMSC for creation of the cellulose films. D.M.H. is a Royal Society Wolfson Fellow.

\section{REFERENCES}

(1) Edwards, D. C. Polymer-filler Interactions in Rubber Reinforcement. J. Mater. Sci. 1990, 25, 4175.

(2) Mittal, K. L., Ed.; Polymer Surface Modifications: Relevance to Adhesion; VSP: Utrecht, 1996.

(3) Russel, W. B.; Saville, D. A.; Schowalter, W. R. Colloidal Dispersions; Cambridge University Press: Cambridge, 1989.

(4) Wicks, Z. W.; Jones, F. N.; Pappas, S. P. Organic Coatings: Science and Technology, 2nd ed.; Wiley-Interscience: New York, 1999.

(5) Fleer, G. J.; Stuart Cohen, M. A.; Scheutjens, J. H.; Cosgrove, T.; Vincent, B. Polymers at Interfaces; Chapman and Hall: London, 1993.

(6) Garg, A.; Heflin, J. R.; Gibson, H. W.; Davis, R. M. Study of Film Structure and Adsorption Kinetics of Polyelectrolyte Multilayer Films: Effect of $\mathrm{pH}$ and Polymer Concentration. Langmuir 2008, 24, 10887.

(7) Plunkett, M. A.; Claesson, P. M.; Ernstsson, M.; Rutland, M. W. Comparison of the adsorption of different charge density polyelectrolytes: A quartz crystal microbalance and X-ray photoelectron spectroscopy study. Langmuir 2003, 19, 4673.

(8) Xu, H.; Schlenoff, J. B. Kinetics, isotherms, and competition in polymer adsorption using the quartz crystal microbalance. Langmuir 1994, 10, 241.

(9) Awan, M. A.; Dimonie, V. L.; Filippov, L. K.; El-Aasser, M. S. Adsorption Kinetics of Amphipathic Polystyrene-block-polybutadiene onto Silicon Wafer and Polystyrene Planar Surfaces. Langmuir 1997, 13, 130.

(10) Bain, C. D. Studies of adsorption at interfaces by optical techniques: ellipsometry, second harmonic generation and sumfrequency generation. Curr. Opin. Colloid Interface Sci. 1998, 3, 287.

(11) Chen, Z.; Shen, Y. R.; Somorjai, G. A. Studies of polymer surfaces by sum frequency generation vibrational spectroscopy. Annu. Rev. Phys. Chem. 2002, 53, 437.

(12) Noskov, B. A.; Akentiev, A. V.; Grigoriev, D. O.; Loglio, G.; Miller, R. Ellipsometric study of nonionic polymer solutions. J. Colloid Interface Sci. 2005, 282, 38.

(13) Vidal, F.; Tadjeddine, A. Sum-frequency generation spectroscopy of interfaces. Rep. Prog. Phys. 2005, 68, 1095.

(14) Walter, H.; Harrats, C.; Muller-Buschbaum, P.; Jerome, R.; Stamm, M. Adsorption of ampholytic diblock copolymers from dilute aqueous solution at the solid/liquid interface. Langmuir 1999, 15, 1260.

(15) Fu, Z. G.; Santore, M. M. Poly(ethylene oxide) adsorption onto chemically etched silicates by Brewster angle reflectivity. Colloids Surf., A 1998, 135, 63.

(16) O'Shaughnessy, B.; Vavylonis, D. Non-equilibrium in adsorbed polymer layers. J. Phys.: Condens. Matter 2005, 17, R63.

(17) Pipino, A. C. R.; Hudgens, J. W.; Huie, R. E. Evanescent wave cavity ring-down spectroscopy for probing surface processes. Chem. Phys. Lett. 1997, 280, 104.

(18) Schnippering, M.; Neil, S. R. T.; Mackenzie, S. R.; Unwin, P. R. Evanescent wave cavity-based spectroscopic techniques as probes of interfacial processes. Chem. Soc. Rev. 2011, 40, 207.

(19) van der Sneppen, L.; Ariese, F.; Gooijer, C.; Ubachs, W. LiquidPhase and Evanescent-Wave Cavity Ring-Down Spectroscopy in Analytical Chemistry. Annu. Rev. Anal. Chem. 2009, 2, 13.

(20) Powell, H. V.; Schnippering, M.; Mazurenka, M.; Macpherson, J. V.; Mackenzie, S. R.; Unwin, P. R. Evanescent Wave Cavity RingDown Spectroscopy as a Probe of Interfacial Adsorption: Interaction of Tris(2,2 '-bipyridine)ruthenium(II) with Silica Surfaces and Polyelectrolyte Films. Langmuir 2009, 25, 248.

(21) Fisk, J. D.; Batten, R.; Jones, G.; O’Reilly, J. P.; Shaw, A. M. pH dependence of the crystal violet adsorption isotherm at the silica-water interface. J. Phys. Chem. B 2005, 109, 14475.

(22) O'Reilly, J. P.; Butts, C. P.; I'Anson, I. A.; Shaw, A. M. Interfacial $\mathrm{pH}$ at an isolated silica-water surface. J. Am. Chem. Soc. 2005, 127, 1632.

(23) Shaw, A. M.; Hannon, T. E.; Li, F. P.; Zare, R. N. Adsorption of crystal violet to the silica-water interface monitored by evanescent wave cavity ring-down spectroscopy. J. Phys. Chem. B 2003, 107, 7070. 
(24) Everest, M. A.; Black, V. M.; Haehlen, A. S.; Haveman, G. A.; Kliewer, C. J.; Neill, H. A. Hemoglobin adsorption to silica monitored with polarization-dependent evanescent-wave cavity ring-down Spectroscopy. J. Phys. Chem. B 2006, 110, 19461.

(25) Fan, H. F.; Hung, C. Y.; Lin, K. C. Molecular adsorption at silica/CH3 $\mathrm{CN}$ interface probed by using evanescent wave cavity ringdown absorption spectroscopy: Determination of thermodynamic properties. Anal. Chem. 2006, 78, 3583.

(26) Schnippering, M.; Unwin, P. R.; Hult, J.; Laurila, T.; Kaminski, C. F.; Langridge, J. M.; Jones, R. L.; Mazurenka, M.; Mackenzie, S. R. Evanescent wave broadband cavity enhanced absorption spectroscopy using supercontinuum radiation: A new probe of electrochemical processes. Electrochem. Commun. 2008, 10, 1827.

(27) Fan, H. F.; Li, F.; Zare, R. N.; Lin, K. C. Characterization of two types of silanol groups on fused-silica surfaces using evanescent-wave cavity ring-down spectroscopy. Anal. Chem. 2007, 79, 3654.

(28) Li, F. P.; Zare, R. N. Molecular orientation study of methylene blue at an air/fused-silica interface using evanescent-wave cavity ringdown spectroscopy. J. Phys. Chem. B 2005, 109, 3330.

(29) Chen, M. S.; Fan, H. F.; Lin, K.-C. Kinetic and Thermodynamic Investigation of Rhodamine B Adsorption at Solid/Solvent Interfaces by Use of Evanescent-Wave Cavity Ring-Down Spectroscopy. Anal. Chem. 2010, 82, 868.

(30) Schnippering, M.; Powell, H. V.; Zhang, M. Q.; Macpherson, J. V.; Unwin, P. R.; Mazurenka, M.; Mackenzie, S. R. Surface assembly and redox dissolution of silver nanoparticles monitored by evanescent wave cavity ring-down spectroscopy. J. Phys. Chem. C 2008, 112, 15274.

(31) Schnippering, M.; Powell, H. V.; Mackenzie, S. R.; Unwin, P. R. Real-Time Monitoring of Polyaniline Nanoparticle Formation on Surfaces. J. Phys. Chem. C 2009, 113, 20221.

(32) Martin, W. B.; Mirov, S.; Martyshkin, D.; Venugopalan, R.; Shaw, A. M. Hemoglobin adsorption isotherm at the silica-water interface with evanescent wave cavity ring-down spectroscopy. $J$. Biomed. Opt. 2005, 10.

(33) Powell, H. V.; O'Connell, M. A.; Zhang, M.; Mackenzie, S. R.; Unwin, P. R. Evanescent Wave Cavity Ringdown Spectroscopy (EWCRDS): A Platform for the Study of Supported Lipid Bilayers. Anal. Chem. 2012, 84, 2585.

(34) Powell, H. V.; Schnippering, M.; Cheung, M.; Macpherson, J. V.; Mackenzie, S. R.; Stavros, V. G.; Unwin, P. R. Probing Redox Reactions of Immobilized Cytochrome c Using Evanescent Wave Cavity Ring-Down Spectroscopy in a Thin-Layer Electrochemical Cell. ChemPhysChem 2010, 11, 2985.

(35) Wang, X. F.; Hinz, M.; Vogelsang, M.; Welsch, T.; Kaufmann, D.; Jones, H. A new approach to detecting biologically active substances with evanescent-wave cavity ring-down spectroscopy. Chem. Phys. Lett. 2008, 467, 9.

(36) Zhang, M. Q.; Powell, H. V.; Mackenzie, S. R.; Unwin, P. R. Kinetics of Porphyrin Adsorption and DNA-Assisted Desorption at the Silica-Water Interface. Langmuir 2010, 26, 4004.

(37) Ong, S. W.; Zhao, X. L.; Eisenthal, K. B. Polarization of WaterMolecules at a Charged Interface - 2nd Harmonic Studies of the Silica Water Interface. Chem. Phys. Lett. 1992, 191, 327.

(38) Dong, Y.; Pappu, S. V.; Xu, Z. Detection of local density distribution of isolated silanol groups on planar silica surfaces using nonlinear optical molecular probes. Anal. Chem. 1998, 70, 4730.

(39) Haselberg, R.; van der Sneppen, L.; Ariese, F.; Ubachs, W.; Gooijer, C.; de Jong, G. J.; Somsen, G. W. Effectiveness of Charged Noncovalent Polymer Coatings against Protein Adsorption to Silica Surfaces Studied by Evanescent-Wave Cavity Ring-Down Spectroscopy and Capillary Electrophoresis. Anal. Chem. 2009, 81, 10172.

(40) van der Sneppen, L.; Gooijer, C.; Ubachs, W.; Ariese, F. Evanescent-wave cavity ring-down detection of cytochrome $c$ on surface-modified prisms. Sens. Actuators, B 2009, 139, 505.

(41) Kontturi, E.; Tammelin, T.; Osterberg, M. Cellulose - model films and the fundamental approach. Chem. Soc. Rev. 2006, 35, 1287.
(42) Klemm, D.; Heublein, B.; Fink, H.-P.; Bohn, A. Cellulose: Fascinating Biopolymer and Sustainable Raw Material. Angew. Chem., Int. Ed. 2005, 44, 3358.

(43) Jones, M. W.; Gibson, M. I.; Mantovani, G.; Haddleton, D. M. Tunable thermo-responsive polymer-protein conjugates via a combination of nucleophilic thiol-ene "click" and SET-LRP. Polym. Chem. 2011, 2, 572.

(44) Levere, M. E.; Willoughby, I.; O’Donohue, S.; de Cuendias, A.; Grice, A. J.; Fidge, C.; Becer, C. R.; Haddleton, D. M. Assessment of SET-LRP in DMSO using online monitoring and Rapid GPC. Polym. Chem. 2010, 1, 1086.

(45) Nguyen, N. H.; Percec, V. SET-LRP of Methyl Acrylate Catalyzed with Activated $\mathrm{Cu}(0)$ Wire in Methanol in the Presence of Air. J. Polym. Sci., Part A1 2011, 49, 4756.

(46) Percec, V.; Guliashvili, T.; Ladislaw, J. S.; Wistrand, A.; Stjerndahl, A.; Sienkowska, M. J.; Monteiro, M. J.; Sahoo, S. Ultrafast synthesis of ultrahigh molar mass polymers by metal-catalyzed living radical polymerization of acrylates, methacrylates, and vinyl chloride mediated by SET at 25 degrees C. J. Am. Chem. Soc. 2006, 128, 14156.

(47) Soeriyadi, A. H.; Boyer, C.; Nystroem, F.; Zetterlund, P. B.; Whittaker, M. R. High-Order Multiblock Copolymers via Iterative $\mathrm{Cu}(0)$-Mediated Radical Polymerizations (SET-LRP): Toward Biological Precision. J. Am. Chem. Soc. 2011, 133, 11128.

(48) Geng, J.; Mantovani, G.; Tao, L.; Nicolas, J.; Chen, G.; Wallis, R.; Mitchell, D. A.; Johnson, B. R. G.; Evans, S. D.; Haddleton, D. M. Site-directed conjugation of "Clicked" glycopolymers to form glycoprotein mimics: Binding to mammalian lectin and induction of immunological function. J. Am. Chem. Soc. 2007, 129, 15156.

(49) Mazurenka, M.; Hamilton, S. M.; Unwin, P. R.; Mackenzie, S. R. In-situ measurement of colloidal gold adsorption on functionalized silica surfaces. J. Phys. Chem. C 2008, 112, 6462.

(50) Mazurenka, M.; Wada, R; Shillings, A. J. L.; Butler, T. J. A.; Beames, J. M.; Orr-Ewing, A. Fast Fourier transform analysis in cavity ring-down spectroscopy: application to an optical detector for atmospheric NO2. J. Appl. Phys., B 2005, 81, 135.

(51) Mazurenka, M.; Wilkins, L.; Macpherson, J. V.; Unwin, P. R.; Mackenzie, S. R. Evanescent wave cavity ring-down spectroscopy in a thin-layer electrochemical cell. Anal. Chem. 2006, 78, 6833.

(52) Schaub, M.; Wenz, G.; Wegner, G.; Stein, A.; Klemm, D. Ultrathin Films of Cellulose on Silicon-Wafers. Adv. Mater. 1993, 5, 919.

(53) Rehfeldt, F.; Tanaka, M. Hydration forces in ultrathin films of cellulose. Langmuir 2003, 19, 1467.

(54) Tanaka, M.; Kaufmann, S.; Nissen, J.; Hochrein, M. Orientation selective immobilization of human erythrocyte membranes on ultrathin cellulose films. Phys. Chem. Chem. Phys. 2001, 3, 4091. 